\title{
Experimental estimation of the quantum Fisher information from randomized measurements
}

\author{
Min Yu $\odot,{ }^{1, *}$ Dongxiao Li $\odot,{ }^{1, *}, * *$ Jingcheng Wang, ${ }^{1}$ Yaoming Chu $\odot,{ }^{1}$ Pengcheng Yang, ${ }^{1}$ Musang Gong, ${ }^{1}$ \\ Nathan Goldman, ${ }^{2, \dagger}$ and Jianming Cai $\odot^{1,3,4, \sharp}$ \\ ${ }^{1}$ School of Physics, International Joint Laboratory on Quantum Sensing and Quantum Metrology, \\ Huazhong University of Science and Technology, Wuhan 430074, China \\ ${ }^{2}$ Center for Nonlinear Phenomena and Complex Systems, Université Libre de Bruxelles, CP 231, Campus Plaine, B-1050 Brussels, Belgium \\ ${ }^{3}$ Wuhan National High Magnetic Field Center, Huazhong University of Science and Technology, Wuhan 430074, China \\ ${ }^{4}$ State Key Laboratory of Precision Spectroscopy, East China Normal University, Shanghai 200062, China
}

(Received 2 April 2021; accepted 2 November 2021; published 16 November 2021)

\begin{abstract}
The quantum Fisher information (QFI) represents a fundamental concept in quantum physics. It quantifies the metrological potential of quantum states in quantum parameter estimation measurements, and is intrinsically related to quantum geometry and multipartite entanglement of many-body systems. Using a nitrogen-vacancy center spin in diamond, we experimentally demonstrate a randomized-measurement method to extract the QFI of the qubit, for both pure and mixed states. We then apply this scheme to a 4-qubit state, using a superconducting quantum computer, and show that it provides access to the sub-QFI, which sets a lower bound on the QFI for general mixed states. We numerically study the scaling of statistical error, considering $N$-qubit states, to illustrate the advantage of our randomized-measurement approach in estimating the QFI and multipartite entanglement. Our results highlight the general applicability of our method to different quantum platforms, including solid-state spin systems, superconducting quantum computers, and trapped ions.
\end{abstract}

DOI: 10.1103/PhysRevResearch.3.043122

\section{INTRODUCTION}

Quantum technologies promise appealing advantages in various practical applications. As a prime example, quantum metrology [1] exploits quantum resources such as entanglement [2], coherence [3], squeezing [4], and criticality [5-8] to achieve unprecedented measurement performance. This has applications in a variety of fields, including precision measurements in physics [9-11], material science [12], and biology [13]. The metrological potential of quantum resource states is quantified by the quantum Fisher information (QFI) [14], which is an extension of the Fisher information [15] in the quantum realm. According to the quantum Cramér-Rao bound, the inverse of the QFI sets a fundamental limit on the accuracy of parameter-estimation measurements [16].

Besides its role in quantum metrology, the QFI also represents a fundamental concept in quantum physics. It has been shown to exhibit deep connections with multipartite entanglement [17-20]. From a geometric perspective, the QFI characterizes the distinguishability between neighboring

\footnotetext{
*These authors contributed equally to this work.

***idongxiao414@ hust.edu.cn

†ngoldman@ulb.ac.be

¥jianmingcai@hust.edu.cn
}

Published by the American Physical Society under the terms of the Creative Commons Attribution 4.0 International license. Further distribution of this work must maintain attribution to the author(s) and the published article's title, journal citation, and DOI. quantum states in parametric space, which is closely related to the concept of the quantum geometric tensor [21]. It thus plays a significant role in numerous quantum phenomena [22], including quantum phase transitions [5], quantum Zeno dynamics [23], as well as in a variety of quantum information processing protocols.

Identifying experimental methods to extract the QFI of arbitrary quantum states is an outstanding challenge, which is currently under intense investigation [24-27]. Indeed, the QFI is not a linear function of quantum states and it is not related to any observable that can be directly accessed [14]. In principle, one can obtain the value of the QFI for general quantum states (pure or mixed) based on quantum state tomography. However, the number of required measurements increases rapidly with the system size, hence resulting in exceptionally heavy experimental overheads for many-body quantum systems. One route is provided by dynamical susceptibilities [20], which can be probed spectroscopically $[28,29]$ and give access to the QFI of thermal states. Although it is possible to extract the Fisher information based on the Hellinger distance [24], the experimental determination of the QFI for general states still remains a challenging task as it requires, by definition, the determination of the optimal measurement for which the Fisher information is maximized [14].

In this work, we experimentally explore the possibility of estimating the QFI of spin systems using a randomizedmeasurement approach. In the case of a single qubit, which we experimentally explore using a nitrogen-vacancy (NV) center spin, our method allows for the accurate extraction of the QFI, for both pure and mixed states generated by noise. In the multiqubit case, which we study using a superconducting 
quantum computer (IBM Q), our method gives access to the sub-QFI [30], which is equivalent to the QFI for pure states and sets a lower bound on the QFI for general mixed states. Our scheme only relies on random measurements performed on single copies of the quantum system [31], and it does not require full quantum state tomography. Such a unique feature can significantly relax the experimental requirements on nonlocal operations or the number of measurements. It is worth noting that techniques based on randomized measurements have been exploited in different physical contexts, such as the estimation of the $n$th moment of general quantum states [32], the Rényi entanglement entropy [33,34], the overlap of two mixed states [35], the mixed-state entanglement [36,37], and the many-body Chern number [38].

\section{BASIC PRINCIPLE}

We start by considering the standard scenario of quantum parameter estimation, in which the information of the parameter $\theta$ is encoded in a parameter-dependent quantum state $\rho_{\theta}$. The maximum available information to extract $\theta$ is determined by the QFI, denoted as $\mathcal{F}_{\theta}$. As dictated by the quantum Cramér-Rao bound, the ultimate precision of quantum parameter estimation is given by $\delta \theta=1 / \sqrt{\mathcal{F}_{\theta}}$. For a general quantum state, spectrally decomposed as $\rho_{\theta}=\sum_{\lambda} p_{\lambda}|\lambda\rangle\langle\lambda|$, with $p_{\lambda}$ and $|\lambda\rangle$ its eigenvalues and corresponding eigenstates, the QFI can be written as [39]

$$
\mathcal{F}_{\theta}\left(\rho_{\theta}\right)=\sum_{\lambda, \lambda^{\prime}} \frac{2\left|\left\langle\lambda\left|\partial_{\theta} \rho_{\theta}\right| \lambda^{\prime}\right\rangle\right|^{2}}{p_{\lambda}+p_{\lambda^{\prime}}},
$$

where the sum only includes the terms with $p_{\lambda}+p_{\lambda^{\prime}} \neq 0$. A direct way to measure the QFI would be to fully reconstruct the density matrices $\rho_{\theta}$ and $\rho_{\theta+d \theta}$, by performing quantum state tomography $[26,40]$. However, quantum state tomography requires a rapidly increasing number of measurements as the system dimension grows.

Starting from the superfidelity [41], defined as

$$
g\left(\rho_{1}, \rho_{2}\right)=\left[\operatorname{Tr}\left(\rho_{1} \rho_{2}\right)+\sqrt{\left(1-\operatorname{Tr} \rho_{1}^{2}\right)\left(1-\operatorname{Tr} \rho_{2}^{2}\right)}\right],
$$

one can introduce a quantity $\mathcal{F}_{G}\left(\rho_{\theta}\right)$ that provides a lower bound to the QFI of any general quantum state:

$$
\mathcal{F}_{\theta}\left(\rho_{\theta}\right) \geqslant \mathcal{F}_{G}\left(\rho_{\theta}\right) \equiv \frac{\mathcal{D}_{G}^{2}\left(\rho_{\theta}, \rho_{\theta+d \theta}\right)}{(d \theta)^{2}},
$$

where $\mathcal{D}_{G}^{2}\left(\rho_{1}, \rho_{2}\right)=8\left[1-g^{1 / 2}\left(\rho_{1}, \rho_{2}\right)\right]$ denotes the modified Bures distance between two quantum states $\rho_{1}$ and $\rho_{2}$ [41]. We note that $\mathcal{F}_{G}\left(\rho_{\theta}\right)$ was introduced recently in [30], where it was coined "sub-QFI". Thus, by measuring the superfidelity, one is able to extract the lower bound of the QFI for any general quantum state. We stress that the inequality in Eq. (3) becomes an equality, and hence, the sub-QFI becomes equivalent to the QFI, when the quantum state is pure. Moreover, in the case of a single qubit, QFI and sub-QFI are strictly equivalent for both pure and mixed states, as we will illustrate below using an NV-center spin.

In experiments, we first perform random local measurements on the states $\rho_{\theta}$ and $\rho_{\theta+d \theta}$. This allows us to obtain the values of the state overlap $\operatorname{Tr}\left(\rho_{\theta} \rho_{\theta+d \theta}\right)$ and the state purity $\operatorname{Tr}\left(\rho_{\theta}^{2}\right)$ and $\operatorname{Tr}\left(\rho_{\theta+d \theta}^{2}\right)$, following the methods of Refs. [32-35] and the detailed protocol described in Appendix A. The results provide the modified Bures distance $\mathcal{D}_{G}^{2}\left(\rho_{\theta}, \rho_{\theta+d \theta}\right)$, the polynomial fit to which leads to the value of the sub-QFI $\mathcal{F}_{G}\left(\rho_{\theta}\right)$; see Eq. (3). The approach demonstrated here can be generalized to a collective observable suitable for many-body systems (see Appendix A).

\section{EXPERIMENTAL DEMONSTRATION USING A SOLID-STATE SPIN}

In our experimental study, we first measure the QFI of quantum sensor states encoded by a single nitrogen-vacancy (NV) center spin in diamond [42-44]. By applying an external magnetic field $B_{z}$ along the $\mathrm{NV}$ axis, we lift the degeneracy of the spin states $m_{s}= \pm 1$ of the NV center and encode a qubit using the two spin sublevels $m_{s}=0,-1$ with an energy gap $\omega_{0}=D-\gamma_{e} B_{z}$, where $D=(2 \pi) 2.87 \mathrm{GHz}$ is the zerofield splitting and $\gamma_{e}$ is the electronic gyromagnetic ratio. The external magnetic field is chosen as $B_{z} \simeq 510 \mathrm{G}$ such that the associated nitrogen nuclear spin in proximity to the NV center is polarized by optical pumping. The measurement protocol in our experiment is shown in Fig. 1(a). The NV-center spin is prepared in the state $\left|\psi_{\phi}(0)\right\rangle=\cos (\phi / 2)|0\rangle+\sin (\phi / 2)|-1\rangle$ by applying a microwave pulse $\hat{Y}_{\phi}$ with frequency $\omega$ to rotate the spin around the $\hat{y}$ axis by an angle $\phi$ after the optical initialization. The NV-center spin evolves as

$$
\rho(t)=\left[\begin{array}{cc}
\cos ^{2}(\phi / 2) & \frac{1}{2} \sin (\phi) e^{i \theta-\left(t / T_{2}^{*}\right)^{2}} \\
\frac{1}{2} \sin (\phi) e^{-i \theta-\left(t / T_{2}^{*}\right)^{2}} & \sin ^{2}(\phi / 2)
\end{array}\right],
$$

where $\theta=\Delta t$ with $\Delta=\omega-\left(D-\gamma_{e} B_{z}\right)$ is the unknown parameter, from which one can infer the accurate value of the magnetic field $B_{z}$. The dephasing noise causes the decay of the $\mathrm{NV}$-center spin coherence at a rate of $\left(T_{2}^{*}\right)^{-1}$

In our experiment, the randomized measurement is realized by a spin-dependent fluorescence measurement following three microwave pulses [Fig. 1(a)], which implement a random unitary transformation of the form $U=$ $R_{x}(\alpha) R_{y}(\beta) R_{x}(\gamma)$, where $R_{v}(\varphi)$ denotes the rotation around the $\hat{v}$ axis by an angle $\varphi$. The rotation angles $\alpha, \beta, \gamma$ are chosen in a random way such that the unitary transformation $U$ acting on the NV-center spin belongs to the circular unitary ensemble [33]. The subsequent fluorescence measurement is equivalent to the projective measurement on the state $\rho(t)$ along a random basis $|u\rangle=U^{\dagger}|0\rangle$, the outcome of which is given by $p(t)=\operatorname{Tr}\left[|0\rangle\langle 0| U \rho(t) U^{\dagger}\right]=\langle u|\rho(t)| u\rangle$. From the random measurements, we can obtain the fidelity of the quantum state $\rho(t)$ with respect to the initial state as $F(t)=\operatorname{Tr}[\rho(t) \rho(0)]=6\langle p(t) p(0)\rangle-1$, where $\langle\cdots\rangle$ denotes the average over $n$ random matrices $U$; see Eq. (A12) (Appendix A). The result in Fig. 1(b) shows a good agreement with the standard deterministic projective measurement.

In a similar way, we proceed by performing local measurements on the states $\rho_{\theta}=\rho(t)$ and $\rho_{\theta+d \theta}=\rho(t+d t)$ with $d \theta=$ $\Delta d t$, which allows us to obtain the values of $\operatorname{Tr}\left(\rho_{\theta} \rho_{\theta+d \theta}\right)$, $\operatorname{Tr}\left(\rho_{\theta}^{2}\right)$, and $\operatorname{Tr}\left(\rho_{\theta+d \theta}^{2}\right)$ [32-35]; using Eq. (2), this eventually provides the modified Bures distance $\mathcal{D}_{G}^{2}\left(\rho_{\theta}, \rho_{\theta+d \theta}\right)$. The polynomial fit to $\mathcal{D}_{G}^{2}\left(\rho_{\theta}, \rho_{\theta+d \theta}\right)$ is shown as the dashed line in Fig. 1(c), while the solid line only includes the quadratic term. The good agreement between the two curves indicates 
(a)

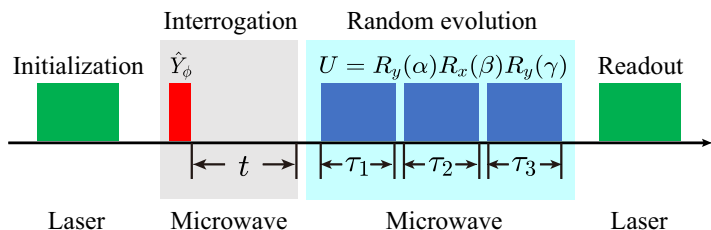

(b)
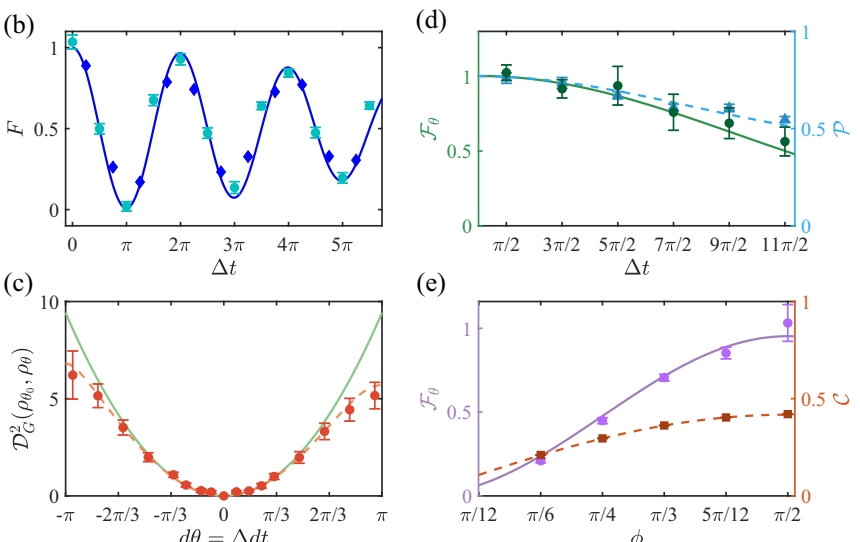

(e)

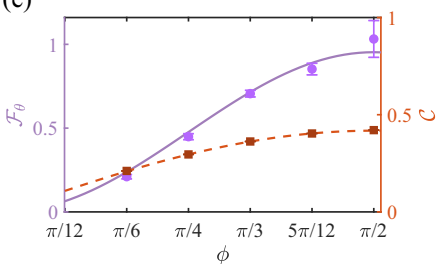

FIG. 1. (a) The pulse sequence for measuring the sub-QFI (which is equivalent to the QFI in the present scenario) in a Ramsey experiment, using an NV-center spin quantum sensor in diamond. The NV-center spin is initially polarized in the state $|0\rangle$ by applying a green laser pulse and the $\phi$-dependent resource state $\left|\psi_{\phi}(0)\right\rangle$ is prepared via a microwave pulse $\hat{Y}_{\phi}$ (i.e., a rotation around the $\hat{y}$ axis by an angle $\phi$ ). The free evolution of duration $t$ results in the parameter-dependent quantum state $\rho_{\theta}=\rho(t)$ with $\theta=\Delta t$; see Eq. (4). The random measurement is implemented by three microwave pulses (blue), with random parameters, followed by spindependent fluorescence measurement. (b) The fidelity between the evolved state $\rho(t)$ and the initial state $\left|\psi_{\phi}(0)\right\rangle$ obtained from randomized measurements (circle) is compared with standard deterministic projective measurement (diamond). (c) The modified Bures distance $\mathcal{D}_{G}^{2}$ between the states $\rho_{\theta_{0}}$ and $\rho_{\theta}$ as a function of $d \theta=\theta-\theta_{0}=$ $\Delta d t$ with $\theta_{0}=3 \pi / 2$. The dashed curve is the polynomial fit to the experiment data (circles), while the solid curve represents the quadratic fit, the coefficient of which provides an estimation for the value of the QFI [see Eq. (3)]. The experiment parameters in (b) and (c) include the detuning $\Delta=(2 \pi) 1.459 \mathrm{MHz}$, the angle $\phi=\pi / 2$, and the number of random measurements $n=400$. The coherence time of the NV-center spin is estimated to be $T_{2}^{*}=2.58 \pm 0.2 \mu \mathrm{s}$. (d) The QFI $\mathcal{F}_{\theta}$ and the purity $\mathcal{P}=\operatorname{Tr}\left[\rho^{2}(t)\right]$ of the evolved state as a function of the free evolution time $t$. The noise causes decoherence and the system evolves into mixed states, which is evidenced by the decrease of the QFI. (e) The QFI $\mathcal{F}_{\theta}$ and the coherence $\mathcal{C}=\left|\rho_{12}(t)\right|$ of the states after a free evolution time $t=3 \pi /(2 \Delta)$ from different initial resource states $\left|\psi_{\phi}(0)\right\rangle=\cos (\phi / 2)|0\rangle+\sin (\phi / 2)|-1\rangle$. The detuning is $\Delta=(2 \pi) 1.459 \mathrm{MHz}$.

the suitable range of $d \theta$ within which the QFI can be extracted from the coefficient of the quadratic term. According to the Taylor expansion of the superfidelity between the states $\rho_{\theta}$ and $\rho_{\theta+d \theta}$, namely $\mathcal{D}_{G}^{2}\left(\rho_{\theta}, \rho_{\theta+d \theta}\right)=\mathcal{F}_{G}\left(\rho_{\theta}\right) d \theta^{2}+O\left(d \theta^{3}\right)$, we can extract $\mathcal{F}_{G}\left(\rho_{\theta}\right)$ from the coefficient of the quadratic term. In the present single-qubit case, the superfidelity is equivalent to the Uhlmann-Jozsa fidelity for both pure and mixed states [41]; we thus obtain the exact QFI through $\mathcal{F}_{\theta}=$ $\mathcal{F}_{G}\left(\rho_{\theta}\right)$; see Eq. (3).

We show in Fig. 1(d) the measured dynamical evolution of the QFI for the state $\rho(t)$. The result shows the influence of dephasing noise on the QFI and thereby the metrological potential of the evolving state. The system becomes mixed as evidenced by the purity $\mathcal{P}=\operatorname{Tr}\left[\rho^{2}(t)\right]$, which is obtained from local random-measurement results on the evolved state $\rho(t)$, following the protocol detailed in Appendix A. We note that the evolution of the QFI may serve as an indicator of the environmental noise properties in open quantum systems, e.g., non-Markovianity [26]. The present technique does not require any prior information on the system and thus provides a powerful tool to investigate general open quantum systems using the concept of the QFI. Furthermore, we measure the QFI of the evolved state $\rho(t)$ for different initial states $\left|\psi_{\phi}(0)\right\rangle=\cos (\phi / 2)|0\rangle+\sin (\phi / 2)|-1\rangle$, to establish the relation between the QFI and the coherence of the resource states [as quantified by the off-diagonal element of the density matrix $\left.\mathcal{C}=\left|\rho_{12}(t)\right|\right]$. From the results displayed in Fig. 1(e), we find that $\rho(t)$ is indeed mixed due to the influence of noise, and that both the QFI and the coherence increase as the initial angle $\phi$ is increased toward $\pi / 2$. The experimental data agree well with the exact value of the QFI, which demonstrates the validity of the present scheme in measuring the QFI for both pure and mixed quantum states.

\section{EXTENSION TO MULTIQUBIT STATES}

As a second demonstration, we generate a 4-qubit GHZ state (with estimated fidelity of 78\%; see Appendix B) and measure the sub-QFI from randomized measurements using the Qiskit by IBM Quantum Experience; the corresponding quantum circuit is shown in Fig. 2(a). We consider the parametrized state $\rho_{\theta}=e^{-i \theta J_{z}} \rho e^{i \theta J_{z}}$, where $J_{z}=$ $\sum_{j=1}^{4} \sigma_{z}^{(j)} / 2$, and apply local random unitary transformations $u_{j}$ from the classical compact group, where $u_{j}$ is determined by the random parameters $\lambda_{j}, \theta_{j}, \varphi_{j}$ through

$$
u_{j}=\left[\begin{array}{cc}
\cos \left(\frac{\theta_{j}}{2}\right) & -e^{i \lambda_{j}} \sin \left(\frac{\theta_{j}}{2}\right) \\
e^{i \varphi_{j}} \sin \left(\frac{\theta_{j}}{2}\right) & e^{i\left(\varphi_{j}+\lambda_{j}\right)} \cos \left(\frac{\theta_{j}}{2}\right)
\end{array}\right] .
$$

The overlap is calculated by the formula $\operatorname{Tr}\left[\rho_{\theta} \rho_{\theta+d \theta}\right]=$ $N \sum_{s, s^{\prime}}(-d)^{D\left[s, s^{\prime}\right]}\left\langle p_{\theta}(s) p_{\theta+d \theta}\left(s^{\prime}\right)\right\rangle[34,35]$ for local random measurement, where $p_{\theta}(s)=\operatorname{Tr}\left[|s\rangle\langle s| U \rho_{\theta} U^{\dagger}\right], d$ is the size of the local Hilbert space, and $D\left[s, s^{\prime}\right]$ is the Hamming distance between states $s, s^{\prime}$. The purity can be also obtained via the similar method. We experimentally determine the modified Bures distance $\mathcal{D}_{G}^{2}\left(\rho_{\theta_{0}}, \rho_{\theta}\right)$ for different values of $\theta$, and perform the polynomial fit correspondingly; see Fig. 2(b). The coefficient of the quadratic term provides the sub-QFI, $\mathcal{F}_{G}\left(\rho_{\theta_{0}}\right)=4.301 \pm 0.22$, which is in good agreement with the value $\mathcal{F}_{G}=4.4807$ obtained from quantum state tomography; see Appendix B. The measured $\mathcal{F}_{G}$ provides the lower bound of the exact QFI, which is $\mathcal{F}_{\theta}=6.5147$ in this case.

We now explore the applicability of our sub-QFI measurement in the context of many-body quantum physics [17], setting the focus on entanglement dynamics in an open $\mathrm{N}$-qubit system. We again consider a GHZ state [45], obtained by applying the unitary operation $U_{N}=$ $\exp \left(i \pi J_{x} / 2\right) \exp \left(i \pi J_{z}^{2} / 2\right) \exp \left(i \pi J_{x} / 2\right)$ on an initialized state $|00 \ldots 0\rangle$; here $J_{\alpha}=\sum_{j=1}^{N} \sigma_{\alpha}^{(j)} / 2$, where $\sigma_{\alpha}^{(j)}$ is the Pauli 
(a)

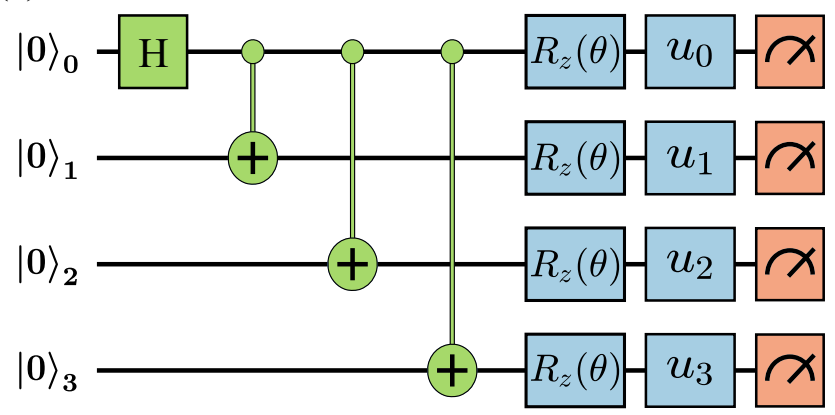

(b)

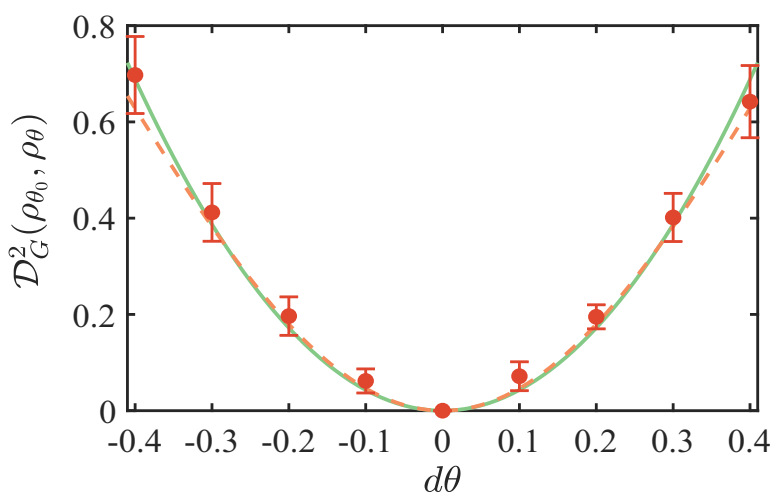

FIG. 2. (a) Quantum circuit for the measurement of the sub-QFI for a four-qubit entangled state. The Hadamard gate and the controlNOT gates are used to prepare the GHZ state, which is used for the estimation of the parameter $\theta$ as induced by the rotation $R_{z}(\theta)$. Local random matrices $u_{j}$ are applied to realize randomized measurements. (b) The modified Bures distance $\mathcal{D}_{G}^{2}$ between the state $\rho_{\theta_{0}}$ and $\rho_{\theta}$ as a function of $d \theta=\theta-\theta_{0}$; here we set $\theta_{0}=0$. The dashed curve is the polynomial fit to the experiment data (circles) generated by the IBM Quantum Falcon Processor (ibmq_belem v1.0.3), while the solid curve represents the quadratic fit. The experimental sub-QFI $\mathcal{F}_{G}=4.301 \pm 0.22$ is compatible with the value $\mathcal{F}_{G}=4.4807$ obtained from quantum state tomography (see Appendix B). This result provides a lower bound for the exact QFI, which is $\mathcal{F}_{\theta}=6.5147$. The number of randomized measurements is $n=400$.

matrix along the $\alpha$ direction of the $j$ th qubit. We describe the dynamical evolution of the system by a quantum master equation, whose dissipator is taken in the form

$$
\mathcal{L} \rho=\sum_{j=1}^{N} L_{j} \rho L_{j}^{\dagger}-\frac{1}{2}\left(L_{j}^{\dagger} L_{j} \rho+\rho L_{j}^{\dagger} L_{j}\right),
$$

where the Lindblad operator $L_{j}=\sqrt{\gamma} \sigma_{z}^{(j)}$ describes the dephasing of the $j$ th qubit with a dephasing rate $\gamma$. To measure the sub-QFI of the mixed state $\rho_{\theta}=e^{-i \theta J_{z}} \rho e^{i \theta J_{z}}$, we design random matrices through the time-evolution operator $U=\prod_{m=1}^{K} e^{-i \mathcal{H}_{m} T}$, where $\mathcal{H}_{m}=\sum_{j=1}^{N} \Delta_{m}^{(j)} \sigma_{z}^{(j)}+H_{s}$. The first term $\Delta_{m}^{(j)} \sigma_{z}^{(j)}$ represents an on-site disorder drawn from a normal distribution with the standard deviation $\delta$, while the second term $H_{s}$ denotes the Ising Hamiltonian that can be implemented in trapped-ion systems [46,47], namely $H_{s}=\sum_{(k<l)} g|k-l|^{-\alpha} \sigma_{x}^{(k)} \sigma_{x}^{(l)}+\Omega \sum_{k=1}^{N} \sigma_{x}^{(k)}$, with $0<\alpha<3$ and $g$ the nearest-neighbor coupling.

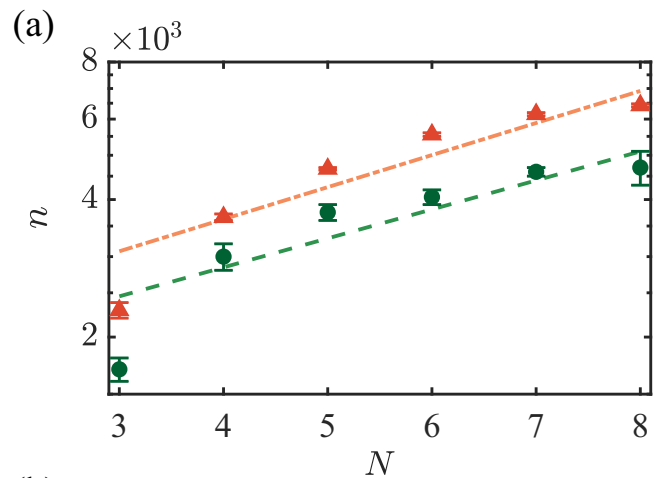

(b)

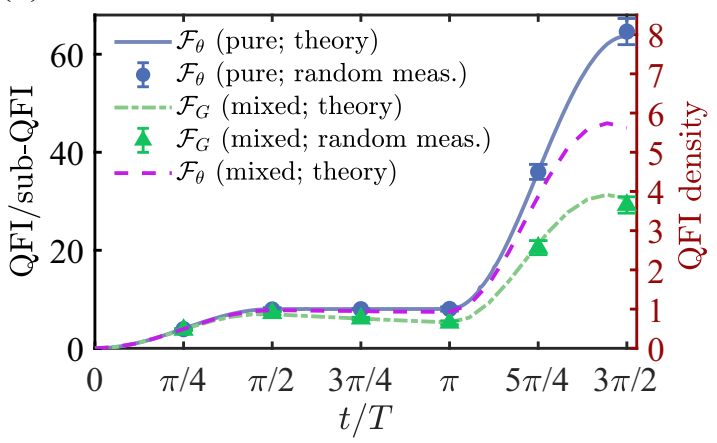

FIG. 3. (a) The number of required measurements $n$ in order to achieve an average relative error $\leqslant \epsilon$ of the QFI (circles, $\gamma=0$ ) and the sub-QFI (triangles, $\gamma / g=0.01$ ) as functions of the number of the ions. The dashed line and the dash-dotted line are the corresponding exponential fits $2^{a+b N}$ with $a=10.63 \pm 0.93, b=0.21 \pm 0.14$ and $a=10.89 \pm 0.82, b=0.23 \pm 0.12$. The parameters are chosen as $\alpha=1.5, K=20, d \theta=1.6 / N$, and $g=\Omega=\delta=1 / T, \epsilon=9 \%$. (b) The QFI and the QFI density as functions of the evolution time for an 8-ion system (solid line for $\gamma=0$ and dashed line for $\gamma / g=0.01$ ) during the generation of GHZ state. The dash-dotted line shows the lower bound of the QFI and the QFI density as $\gamma / g=0.01$. The circles and the triangles are the results obtained by random measurements. The number of random measurement is $n=1000$; the other parameters are the same as in (a): $K=20, \alpha=1.5$, and $g=\Omega=\delta=1 / T$.

We proceed by performing a scaling analysis of the experimental overhead as the system size increases. By choosing two independent sets of random unitary gates to obtain the purity and the state overlap respectively, we obtain a simple formula for the error propagation; see Eq. (C1) in Appendix C. Figure 3(a) shows the scaling of the number of randomized measurements that are required to achieve an average relative error on the sub-QFI that is smaller than some threshold $\leqslant \epsilon=9 \%$. In the absence of noise $(\gamma=0)$, the system is in a pure state, and the number of required measurements is shown to scale as $\sim 2^{(0.21 \pm 0.14) N}$ (dashed line). We remark that such a scaling represents a significant improvement over quantum state tomography, for which the number of required measurements scales as $\sim 2^{2 N}$ [48]. The improvement is also evident for the mixed states generated by noise. In this case, the number of required measurements scales as $\sim 2^{(0.23 \pm 0.12) N}$ (dash-dotted line). Importantly, the QFI is known to serve as a witness of multipartite entanglement $[18,19]$ : a general quantum state $\rho$ must be $(m+1)$-partite entangled if 
the QFI density $\mathcal{F}_{\theta} / N>m$. In Fig. 3(b), we show the QFI (resp. sub-QFI) for pure (resp. mixed) states, together with the corresponding density $\mathcal{F}_{\theta} / N$ as a function of the evolution time. These results demonstrate the efficiency of our scheme in measuring the lower bound of the QFI, and hence the dynamics of multipartite entanglement, in open many-body quantum systems.

\section{CONCLUSIONS AND OUTLOOK}

We used several quantum platforms to demonstrate an efficient method to determine the sub-QFI of general quantum states based on randomized measurements. Our method does not rely on full quantum state tomography, and in fact, it exhibits significantly enhanced efficiency over quantum state tomography when applied to many-body quantum systems. Importantly, the sub-QFI is equivalent to the QFI in single qubits or pure multiqubit states, and it provides an instructive lower bound on the QFI in more general cases. The application of our approach to other quantum systems could provide a powerful method to estimate the QFI in different contexts, allowing for the experimental exploration of the QFI in various quantum phenomena.

Note added in proof. We note a related work on quantum Fisher information from randomized measurement [49].

\section{ACKNOWLEDGMENTS}

We thank Markus Heyl and Tomoki Ozawa for valuable discussions. This work is supported by National Natural Science Foundation of China (Grants No. 11874024, No. 11690032, No. 12047525), the Open Project Program of Wuhan National Laboratory for Optoelectronics (Grant No. 2019WNLOKF002), the Fundamental Research Funds for the Central Universities, and the Interdisciplinary Program of Wuhan National High Magnetic Field Center (Grant No. WHMFC202106). N.G. is supported by the FRS-FNRS (Belgium) and the ERC Starting Grant TopoCold. DL is also funded by China Postdoctoral Science Foundation (Grant No. 2021M690062).

\section{APPENDIX A: EXTRACT QUANTUM STATE INFORMATION VIA GENERAL RANDOM MEASUREMENTS}

The key for the estimation of the sub-QFI in the main text is the measurement of quantum state overlap in order to obtain the superfidelity; see Eqs. (2) and (3). Here, we present the details on how to obtain quantum state overlap by performing random measurements introducing random global unitary gates prior to a projective measurement. Given a random unitary matrix $U$ in the Hilbert space $\mathcal{H}_{N}$ with the dimension $N$, we can obtain the identity

$$
U U^{\dagger}=1
$$

which means that the $m$ th diagonal term of $U U^{\dagger}$ is

$$
\sum_{n=1}^{N} U_{m n} U_{m n}^{*}=1
$$

If $U$ is a random matrix distributed according to the Haar measure, i.e., chosen in the circular unitary ensemble (CUE), one can prove that [32]

$$
\left\langle U_{m n} U_{m n}^{*}\right\rangle=1 / N,
$$

which is a special case of the general result

$$
\left\langle U_{k l} U_{m n}^{*}\right\rangle=\delta_{k m} \delta_{l n} / N .
$$

Here $U_{k l}$ and $U_{m n}^{*}$ are the matrix elements of $U$ and the corresponding complex conjugate respectively. $\langle\cdots\rangle$ denotes the average over the distribution of random unitaries. Higherorder averages follow from the Isserlis ("Gaussian-moments") theorem $[32,33,50]$ including

$$
\begin{aligned}
\left\langle U_{m k} U_{n k}^{*} U_{m^{\prime} k^{\prime}} U_{n^{\prime} k^{\prime}}^{*}\right\rangle= & \frac{1}{N^{2}-1}\left(\delta_{m n} \delta_{m^{\prime} n^{\prime}}+\delta_{k k^{\prime}} \delta_{m n^{\prime}} \delta_{m^{\prime} n}\right) \\
& -\frac{1}{N\left(N^{2}-1\right)}\left(\delta_{m n^{\prime}} \delta_{m^{\prime} n}+\delta_{k k^{\prime}} \delta_{m n} \delta_{m^{\prime} n^{\prime}}\right) .
\end{aligned}
$$

Random measurements can be implemented by first applying random unitary gates which transform the density matrix $\rho_{1}$ as

$$
\mathcal{U}\left(\rho_{1}\right)=U \rho_{1} U^{\dagger}=\sum_{m, n, l, l^{\prime}} \rho_{m n}^{(1)} U_{l m} U_{l^{\prime} n}^{*}|l\rangle\left\langle l^{\prime}\right|,
$$

where $\rho_{m n}^{(1)}$ is the element of $\rho_{1}$, i.e., $\rho_{m n}^{(1)}=\left\langle m\left|\rho_{1}\right| n\right\rangle$, and $\{|n\rangle\}$ forms a basis. The subsequent measurement in a single basis state $|k\rangle \in \mathcal{H}_{N}$ gives the probability $P_{1}(k)$ as

$$
P_{1}(k)=\operatorname{Tr}\left[|k\rangle\langle k| \mathcal{U}\left(\rho_{1}\right)\right]=\sum_{m, n} \rho_{m n}^{(1)} U_{k m} U_{k n}^{*},
$$

the corresponding average of which is thus given by [according to Eq. (A4)]

$$
\left\langle P_{1}(k)\right\rangle=\sum_{m, n} \rho_{m n}^{(1)}\left\langle U_{m k} U_{n k}^{*}\right\rangle=\sum_{m, n} \rho_{m n}^{(1)} \delta_{m n} / N=1 / N,
$$

where we have used the identity $\operatorname{Tr}(\rho)=\sum_{m} \rho_{m m}=1$. Similarly, the probability $P_{2}(k)$ can be obtained by the same random unitary $U$ acting on another density matrix $\rho_{2}$ as

$$
P_{2}(k)=\sum_{m, n} \rho_{m n}^{(2)} U_{k m} U_{k n}^{*}
$$

In order to obtain the overlap between $\rho_{1}$ and $\rho_{2}$, i.e., $\operatorname{Tr}\left[\rho_{1} \rho_{2}\right]$, we further investigate the correlation between $P_{1}(k)$ and $P_{2}(k)$, which is

$$
\left\langle P_{1}(k) P_{2}(k)\right\rangle=\sum_{m, n, m^{\prime}, n^{\prime}} \rho_{m n}^{(1)} \rho_{m^{\prime} n^{\prime}}^{(2)}\left\langle U_{k m} U_{k n}^{*} U_{k m^{\prime}} U_{k n^{\prime}}^{*}\right\rangle .
$$

By making use of the Isserlis theorem in Eq. (A5), the above correlation is found to be

$$
\begin{aligned}
\left\langle P_{1}(k) P_{2}(k)\right\rangle & =\frac{\sum_{m, n} \rho_{m m}^{(1)} \rho_{n n}^{(2)}+\sum_{m, n} \rho_{m n}^{(1)} \rho_{n m}^{(2)}}{N(N+1)} \\
& =\frac{1+\operatorname{Tr}\left(\rho_{1} \rho_{2}\right)}{N(N+1)}
\end{aligned}
$$

which leads to the following result:

$$
\operatorname{Tr}\left(\rho_{1} \rho_{2}\right)=N(N+1)\left\langle P_{1}(k) P_{2}(k)\right\rangle-1 .
$$


Furthermore, one can replace $N$ in Eq. (A12) by the inverse of the average of the probability $1 / P_{1(2)}(k)$ to make the result more accurate when $N$ is a finite value; see Ref. [32]. We note that the result gives the state purity if we choose $\rho_{2}=\rho_{1}$.

In the above discussion, the probability $P_{1}(k)$, see Eq. (A7), is measured in an arbitrary $N$-dimensional basis state $(|k\rangle)$ of the Hilbert space $\mathcal{H}_{N}[32-38,51]$. We remark that it only requires the projective measurement result on one single basis state, which makes it more convenient in both experiment and numerical calculation as compared with the method based on local random unitaries [34,35], which requires the measurement outcome on a complete set of basis states.

It is also worth noting that for a system consisting of $K d$ dimensional objects (i.e., $N=d^{K}$ ), it may be more feasible to measure the probability $P_{1}\left(\hat{s}_{\alpha}\right)$ corresponding to the collective observable $\hat{s}_{\alpha}=\sum_{j=1}^{K}|\alpha\rangle_{j j}\langle\alpha| / K$, where $|\alpha\rangle_{j}$ denotes an arbitrary $d$-dimensional basis state for the $j$ th particle. In this case, we have

$$
P_{1}\left(\hat{s}_{\alpha}\right)=\frac{1}{K} \sum_{j=1}^{K} \operatorname{Tr}\left[|\alpha\rangle_{j j}\langle\alpha| \mathcal{U}\left(\rho_{1}\right)\right] .
$$

For the total system, there will be $N_{A}^{m}=K !(d-1)^{K-m} /$ $[m !(K-m) !]$ eigenstates, in which $m$ particles are in the state $|\alpha\rangle$. Therefore, the probability $P_{1}\left(\hat{s}_{\alpha}\right)$ can be expanded as

$$
P_{1}\left(\hat{s}_{\alpha}\right)=\frac{1}{K} \sum_{m=1}^{K} \sum_{l_{m}=1}^{N_{A}^{m}} m \operatorname{Tr}\left[\left|m, l_{m}\right\rangle\left\langle m, l_{m}\right| \mathcal{U}\left(\rho_{1}\right)\right] .
$$

By making use of the Eq. (A8), the average of $P_{1}\left(\hat{s}_{\alpha}\right)$ over the random unitary gates is given by

$$
\left\langle P_{1}\left(\hat{s}_{\alpha}\right)\right\rangle=\frac{1}{K N} \sum_{m=1}^{K} m N_{A}^{m},
$$

which means the dimension of the total system can be experimentally obtained by

$$
N=\sum_{m=1}^{K} m N_{A}^{m} /\left[K\left\langle P_{1}\left(\hat{s}_{\alpha}\right)\right\rangle\right] .
$$

Then we consider the same random measurement acting on another density matrix $\rho_{2}$ and obtain the probability $P_{2}\left(\hat{s}_{\alpha}\right)$. To calculate the overlap between $\rho_{1}$ and $\rho_{2}$, we can multiply $P_{1}\left(\hat{s}_{\alpha}\right)$ by $P_{2}\left(\hat{s}_{\alpha}\right)$,

$$
P_{1}\left(\hat{s}_{\alpha}\right) P_{2}\left(\hat{s}_{\alpha}\right)=\frac{1}{K^{2}} \sum_{m, m^{\prime}} \sum_{l_{m}, l_{m^{\prime}}} m m^{\prime} \operatorname{Tr}\left[\left|m, l_{m}\right\rangle\left\langle m, l_{m}\right| \mathcal{U}\left(\rho_{1}\right)\right] \operatorname{Tr}\left[\left|m^{\prime}, l_{m^{\prime}}\right\rangle\left\langle m, l_{m^{\prime}}\right| \mathcal{U}\left(\rho_{2}\right)\right] .
$$

Based on Eq. (A5), the corresponding average can be obtained as

$$
\left\langle P_{1}\left(\hat{s}_{\alpha}\right) P_{2}\left(\hat{s}_{\alpha}\right)\right\rangle=c_{1} \frac{1+\operatorname{Tr}\left(\rho_{1} \rho_{2}\right)}{N(N+1)}+c_{2} \frac{N-\operatorname{Tr}\left(\rho_{1} \rho_{2}\right)}{N\left(N^{2}-1\right)},
$$

where

$$
\begin{aligned}
& c_{1}=\frac{1}{K^{2}} \sum_{m} m^{2} N_{A}^{m}, \\
& c_{2}=\frac{1}{K^{2}} \sum_{m}\left[m^{2} N_{A}^{m}\left(N_{A}^{m}-1\right)+\sum_{m^{\prime} \neq m} m m^{\prime} N_{A}^{m} N_{A}^{m^{\prime}}\right] .
\end{aligned}
$$

This leads to the following inverse formula to calculate the overlap between $\rho_{1}$ and $\rho_{2}$ :

$$
\operatorname{Tr}\left(\rho_{1} \rho_{2}\right)=\frac{N\left(N^{2}-1\right)\left\langle P_{1}\left(\hat{s}_{\alpha}\right) P_{2}\left(\hat{s}_{\alpha}\right)\right\rangle+c_{1}-\left(c_{1}+c_{2}\right) N}{c_{1} N-\left(c_{1}+c_{2}\right)} .
$$

In order to verify the above result, we choose the collective observable

$$
\hat{s}_{0}=\frac{1}{8} \sum_{j=1}^{8}|0\rangle_{j j}\langle 0|,
$$

and use Eqs. (2) and (3) in the main text to simulate the subQFI for an 8-ion system in Fig. 4. The parameter-dependent state $\rho_{\theta}$ and the relevant parameters are the same as those of Fig. 3(b) in the main text with $\gamma=0$. The solid line is the theoretical prediction and the circles are the corresponding results obtained via random measurements based on the collective observable $\hat{s}_{\alpha}$. The good agreement between the theoretical description and the random measurement outcomes verifies the validity of our analytical derivation.

\section{APPENDIX B: ADDITIONAL EXPERIMENTAL DATA}

In Fig. 5, we also show the modified Bures distance $\mathcal{D}_{G}^{2}\left(\rho_{\theta_{0}}, \rho_{\theta}\right)$ as functions of $d \theta$ for different values of $\theta_{0}$. The solid, dashed, and dash-dotted curves represent the corresponding quadratic fit of the experiment data, the coefficients

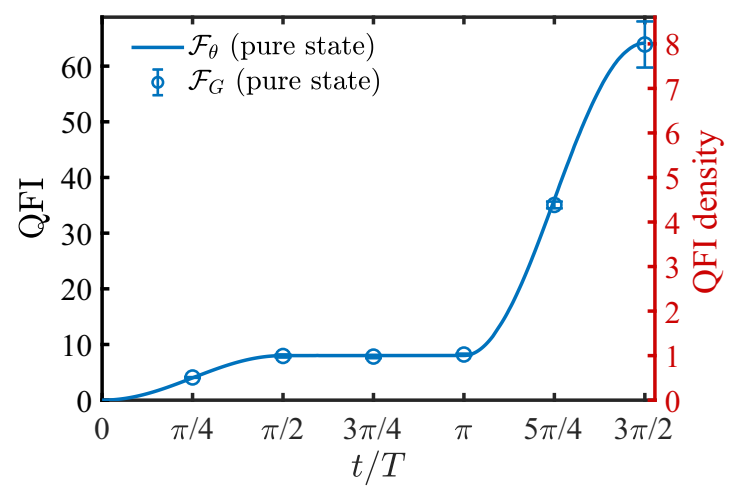

FIG. 4. The QFI and the QFI density obtained from the collective observable $\hat{s}_{0}=\sum_{j=1}^{8}|0\rangle_{j j}\langle 0| / 8$ [see Eq. (A19)] as functions of the evolution time for the same 8-ion system as that of Fig. 3(b) in the main text. The relevant parameters are chosen as $\gamma=0, d \theta=0.1$, $n=2000, K=20, \alpha=1.5$, and $g=\Omega=\delta=1 / T$. 


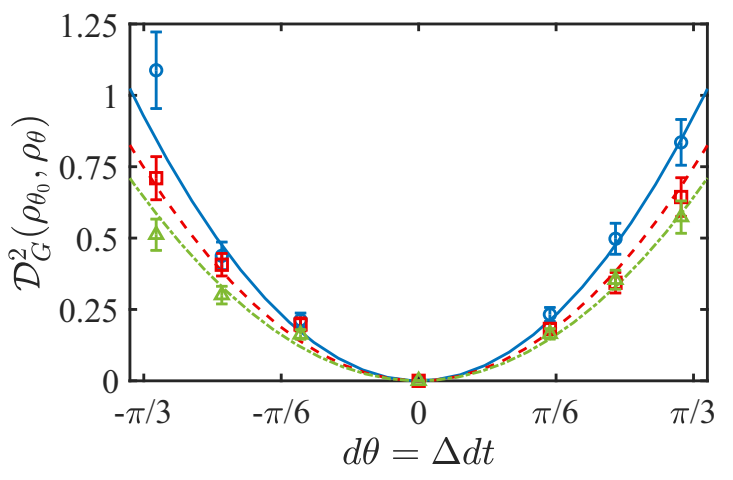

FIG. 5. The modified Bures distance $\mathcal{D}_{G}^{2}\left(\rho_{\theta_{0}}, \rho_{\theta}\right)$ between the states $\rho_{\theta_{0}}$ and $\rho_{\theta}$ as a function of $d \theta=\theta-\theta_{0}=\Delta d t$ for $\theta_{0}=5 \pi / 2$ (circles), $9 \pi / 2$ (squares), and $11 \pi / 2$ (triangles). The solid, dashed, dash-dotted curves are the quadratic fit of the experiment data, the corresponding coefficients of which provide the values of the QFI as $0.94 \pm 0.13,0.69 \pm 0.10$, and $0.56 \pm 0.10$. The other relevant experimental parameters are the detuning $\Delta=(2 \pi) 1.459 \mathrm{MHz}$, the angle $\phi=\pi / 2$, and the number of random measurements is $n=$ 400. The coherence time of the NV-center spin is estimated to be $T_{2}^{*}=2.58 \pm 0.2 \mu \mathrm{s}$. We note that the experimental operations are the same as those of Fig. 1 in the main text.

of which provide estimations for the sub-QFI. Moreover, the values of the sub-QFI that are extracted in this way correspond to the circles of Fig. 1(d) in the main text.

We generate a 4-qubit GHZ state with the IBM Quantum Falcon Processor (ibmq_belem v1.0.3) using the circuit as shown in Fig. 2(a) of the main text. Due to the imperfection of the gates, the prepared state is not an ideal pure GHZ state. Instead, the state is a mixed state. We perform full quantum state tomography, and the entries of the corresponding density matrix that we obtain are shown in Fig. 6. This allows us to estimate that the fidelity of the prepared state with respect to the 4-qubit GHZ state reaches $78 \%$. Based on the density matrix obtained from quantum state tomography, we calculate the parameter-dependent evolution $\rho_{\theta_{0}}=e^{-i \theta_{0} J_{z}} \rho e^{i \theta_{0} J_{z}}$ and $\rho_{\theta}=$ $e^{-i \theta J_{z}} \rho e^{i \theta J_{z}}$, where $J_{z}=\sum_{j=1}^{4} \sigma_{z}^{(j)} / 2$ and $d \theta=\theta-\theta_{0}$. Thus, we can obtain the modified Bures distance $\mathcal{D}_{G}^{2}\left(\rho_{\theta_{0}}, \rho_{\theta}\right)$ with $d \theta=0, \pm 0.1, \pm 0.2, \pm 0.3$ and perform the quadratic fit of $\mathcal{D}_{G}^{2}\left(\rho_{\theta_{0}}, \rho_{\theta}\right) \simeq \mathcal{F}_{G}\left(\rho_{\theta_{0}}\right) d \theta^{2}$ to get the theoretical value of the lower bound of the QFI [following Eqs. (2) and (3) in the main text]. Similarly, by calculating the Uhlmann-Jozsa fidelity be-

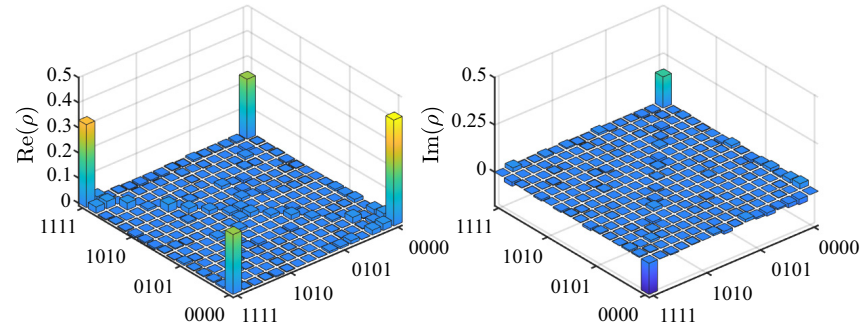

FIG. 6. Density matrix elements of the prepared four-qubit state $\rho$ obtained via Qiskit using the IBM Quantum Falcon Processor (ibmq_belem v1.0.3). The fidelity with respect to the GHZ state is estimated to be $78 \%$.

tween $\rho_{\theta_{0}}$ and $\rho_{\theta}$ we can obtain the exact value of the QFI $\mathcal{F}_{\theta}=6.5147$.

\section{APPENDIX C: SCALING OF STATISTICAL ERROR IN SUB-QFI}

From a statistical viewpoint, $\mathcal{F}_{G}\left(\rho_{\theta}\right)$ in Eqs. (2) and (3) is a function of $\operatorname{Tr} \rho_{\theta}^{2}, \operatorname{Tr} \rho_{\theta+d \theta}^{2}, \operatorname{Tr}\left(\rho_{\theta} \rho_{\theta+d \theta}\right)$. To simplify the analysis, we assume different sets of unitaries to obtain these three quantities via random measurements. In Fig. 3(a) of the main text, the random unitary matrices were generated using random quenching. The uncertainty of sub-QFI becomes

$$
\begin{aligned}
\sigma_{\mathcal{F}_{G}}^{2}= & \left(\frac{\partial \mathcal{F}_{G}}{\partial \operatorname{Tr}\left(\rho_{\theta} \rho_{\theta+d \theta}\right)} \sigma_{\operatorname{Tr}\left(\rho_{\theta} \rho_{\theta+d \theta}\right)}\right)^{2} \\
& +\left(\frac{\partial \mathcal{F}_{G}}{\partial \operatorname{Tr} \rho_{\theta}^{2}} \sigma_{\operatorname{Tr} \rho_{\theta}^{2}}\right)^{2}+\left(\frac{\partial \mathcal{F}_{G}}{\partial \operatorname{Tr} \rho_{\theta+d \theta}^{2}} \sigma_{\operatorname{Tr} \rho_{\theta+d \theta}^{2}}\right)^{2} .
\end{aligned}
$$

Based on the previous analysis in Refs. [51,52], we can treat the estimators as random variables obeying a normal distribution, i.e., $\operatorname{Tr}\left(\rho_{1}^{2}\right) \sim \mathcal{N}\left(\mu_{1}, \sigma_{1}\right), \operatorname{Tr}\left(\rho_{2}^{2}\right) \sim \mathcal{N}\left(\mu_{2}, \sigma_{2}\right)$, $\operatorname{Tr}\left(\rho_{1} \rho_{2}\right) \sim \mathcal{N}\left(\mu_{12}, \sigma_{12}\right)$. Therefore, we can obtain numerical scaling of statistical error for the sub-QFI following Eq. (C1). In Table I, we compare the relative standard deviation (i.e., the ratio between the standard deviation and the average value of the sub-QFI) obtained from the above theoretical analysis and the result from numerical simulation [as shown in Fig. 3(a) of the main text], which demonstrates that the theoretical analysis gives results very similar to those of our numerical simulation.

\begin{tabular}{|c|c|c|c|c|c|c|}
\hline & \multicolumn{6}{|c|}{ Number of qubits } \\
\hline & 3 & 4 & 5 & 6 & 7 & 8 \\
\hline \multicolumn{7}{|l|}{ Pure state: } \\
\hline Theoretical analysis $(\%)$ & 9.2 & 8.6 & 8.6 & 8.7 & 8.5 & 8.4 \\
\hline Numerical simulation (\%) & 9.7 & 9.0 & 9.0 & 9.1 & 8.9 & 8.7 \\
\hline Number of random measurements & $0.85 \times 10^{3}$ & $1.50 \times 10^{3}$ & $1.85 \times 10^{3}$ & $2.05 \times 10^{3}$ & $2.25 \times 10^{3}$ & $2.35 \times 10^{3}$ \\
\hline \multicolumn{7}{|l|}{ Mixed state: } \\
\hline Theoretical analysis $(\%)$ & 9.0 & 9.0 & 8.9 & 8.9 & 8.9 & 9.0 \\
\hline Numerical simulation (\%) & 9.0 & 9.0 & 8.8 & 8.9 & 8.9 & 9.0 \\
\hline Number of random measurements & $1.12 \times 10^{3}$ & $1.86 \times 10^{3}$ & $2.33 \times 10^{3}$ & $2.78 \times 10^{3}$ & $3.06 \times 10^{3}$ & $3.24 \times 10^{3}$ \\
\hline
\end{tabular}

TABLE I. Relative standard deviation of the sub-QFI. 
[1] V. Giovannetti, S. Lloyd, and L. Maccone, Advances in quantum metrology, Nat. Photon. 5, 222 (2011).

[2] V. Giovannetti, S. Lloyd, and L. Maccone, Quantum Metrology, Phys. Rev. Lett. 96, 010401 (2006).

[3] D. P. Pires, I. A. Silva, E. R. deAzevedo, D. O. Soares-Pinto, and J. G. Filgueiras, Coherence orders, decoherence, and quantum metrology, Phys. Rev. A 98, 032101 (2018).

[4] L. Maccone and A. Riccardi, Squeezing metrology: A unified framework, Quantum 4, 292 (2020).

[5] P. Zanardi, M. G. A. Paris, and L. Campos Venuti, Quantum criticality as a resource for quantum estimation, Phys. Rev. A 78, 042105 (2008).

[6] M. M. Rams, P. Sierant, O. Dutta, P. Horodecki, and J. Zakrzewski, At the Limits of Criticality-Based Quantum Metrology: Apparent Super-Heisenberg Scaling Revisited, Phys. Rev. X 8, 021022 (2018).

[7] Y. Chu, S. Zhang, B. Yu, and J. Cai, Dynamic Framework for Criticality-Enhanced Quantum Sensing, Phys. Rev. Lett. 126, 010502 (2021).

[8] I. Frérot and T. Roscilde, Quantum Critical Metrology, Phys. Rev. Lett. 121, 020402 (2018).

[9] B. P. Abbott et al. (LIGO Scientific Collaboration and Virgo Collaboration), GW151226: Observation of Gravitational Waves from a 22-Solar-Mass Binary Black Hole Coalescence, Phys. Rev. Lett. 116, 241103 (2016).

[10] C. Chin, V. Leiber, V. Vuletić, A. J. Kerman, and S. Chu, Measurement of an electron's electric dipole moment using Cs atoms trapped in optical lattices, Phys. Rev. A 63, 033401 (2001).

[11] C. J. Berglund, L. R. Hunter, D. Krause, Jr., E. O. Prigge, M. S. Ronfeldt, and S. K. Lamoreaux, New Limits on Local Lorentz Invariance from $\mathrm{Hg}$ and Cs Magnetometers, Phys. Rev. Lett. 75, 1879 (1995).

[12] F. Casola, T. van der Sar, and A. Yacoby, Probing condensed matter physics with magnetometry based on nitrogen-vacancy centres in diamond, Nat. Rev. Mater. 3, 17088 (2018).

[13] G. Kucsko, P. C. Maurer, N. Y. Yao, M. Kubo, H. J. Noh, P. K. Lo, H. Park, and M. D. Lukin, Nanometre-scale thermometry in a living cell, Nature (London) 500, 54 (2013).

[14] S. L. Braunstein, C. M. Caves, and G. J. Milburn, Generalized uncertainty relations: Theory, examples, and Lorentz invariance, Ann. Phys. 247, 135 (1996).

[15] R. A. Fisher, Theory of statistical estimation, Proc. Cambridge Philos. Soc. 22, 700 (1925).

[16] M. G. A. Paris, Quantum estimation for quantum technology, Int. J. Quantum Inf. 7, 125 (2009).

[17] L. Pezzé and A. Smerzi, Entanglement, Nonlinear Dynamics, and the Heisenberg Limit, Phys. Rev. Lett. 102, 100401 (2009).

[18] P. Hyllus, W. Laskowski, R. Krischek, C. Schwemmer, W. Wieczorek, H. Weinfurter, L. Pezzé, and A. Smerzi, Fisher information and multiparticle entanglement, Phys. Rev. A 85, 022321 (2012).

[19] G. Tóth, Multipartite entanglement and high-precision metrology, Phys. Rev. A 85, 022322 (2012).

[20] P. Hauke, M. Heyl, L. Tagliacozzo, and P. Zoller, Measuring multipartite entanglement through dynamic susceptibilities, Nat. Phys. 12, 778 (2016).

[21] M. Kolodrubetz, D. Sels, P. Mehta, and A. Polkovnikov, Geometry and non-adiabatic response in quantum and classical systems, Phys. Rep. 697, 1 (2017).
[22] L. Pezzè, A. Smerzi, M. K. Oberthaler, R. Schmied, and P. Treutlein, Quantum metrology with nonclassical states of atomic ensembles, Rev. Mod. Phys. 90, 035005 (2018).

[23] A. Smerzi, Zeno Dynamics, Indistinguishability of State, and Entanglement, Phys. Rev. Lett. 109, 150410 (2012).

[24] H. Strobel, W. Muessel, D. Linnemann, T. Zibold, D. B. Hume, L. Pezzè, A. Smerzi, and M. K. Oberthaler, Fisher information and entanglement of non-Gaussian spin states, Science 345, 424 (2014).

[25] F. Fröwis, P. Sekatski, and W. Dür, Detecting Large Quantum Fisher Information with Finite Measurement Precision, Phys. Rev. Lett. 116, 090801 (2016).

[26] Y.-N. Lu, Y.-R. Zhang, G.-Q. Liu, F. Nori, H. Fan, and X.-Y. Pan, Observing Information Backflow from Controllable NonMarkovian Multichannels in Diamond, Phys. Rev. Lett. 124, 210502 (2020).

[27] M. Yu, Y. Liu, P. Yang, M. Gong, Q. Cao, S. Zhang, H. Liu, M Heyl, T. Ozawa, N. Goldman, and J. Cai, Saturating the quantum Cramér-Rao bound and measuring the related quantum Fisher information in a nitrogen-vacancy center in diamond, arXiv:2003.08373.

[28] T. Ozawa and N. Goldman, Extracting the quantum metric tensor through periodic driving, Phys. Rev. B 97, 201117(R) (2018).

[29] T. Ozawa and N. Goldman, Probing localization and quantum geometry by spectroscopy, Phys. Rev. Research 1, 032019(R) (2019).

[30] M. Cerezo, A. Sone, J. L. Beckey, and P. J. Coles, Sub-quantum Fisher information, Quantum Sci. Technol. 6, 035008 (2021).

[31] C. Zhang, B. Yadin, Z.-B. Hou, H. Cao, B.-H. Liu, Y.-F. Huang, R. Maity, V. Vedral, C.-F. Li, G.-C. Guo, and D. Girolami, Detecting metrologically useful asymmetry and entanglement by a few local measurements, Phys. Rev. A 96, 042327 (2017).

[32] S. J. van Enk and C. W. J. Beenakker, Measuring $\operatorname{Tr} \rho^{n}$ on Single Copies of $\rho$ Using Random Measurements, Phys. Rev. Lett. 108, 110503 (2012).

[33] A. Elben, B. Vermersch, M. Dalmonte, J. I. Cirac, and P. Zoller, Rényi Entropies from Random Quenches in Atomic Hubbard and Spin Models, Phys. Rev. Lett. 120, 050406 (2018).

[34] T. Brydges, A. Elben, P. Jurcevic, B. Vermersch, C. Maier, B. P Lanyon, P. Zoller, R. Blatt, and C. F. Roos, Probing Rényi entanglement entropy via randomized measurements, Science 364, 260 (2019).

[35] A. Elben, B. Vermersch, R. van Bijnen, C. Kokail, T. Brydges, C. Maier, M. K. Joshi, R. Blatt, C. F. Roos, and P. Zoller, CrossPlatform Verification of Intermediate Scale Quantum Devices, Phys. Rev. Lett. 124, 010504 (2020).

[36] A. Elben, R. Kueng, H.-Y. R. Huang, R. van Bijnen, C. Kokail, M. Dalmonte, P. Calabrese, B. Kraus, J. Preskill, P. Zoller, and B. Vermersch, Mixed-State Entanglement from Local Randomized Measurements, Phys. Rev. Lett. 125, 200501 (2020).

[37] Y. Zhou, P. Zeng, and Z. Liu, Single-Copies Estimation of Entanglement Negativity, Phys. Rev. Lett. 125, 200502 (2020).

[38] Z.-P. Cian, H. Dehghani, A. Elben, B. Vermersch, G. Zhu, M Barkeshli, P. Zoller, and M. Hafezi, Many-Body Chern Number from Statistical Correlations of Randomized Measurements, Phys. Rev. Lett. 126, 050501 (2021).

[39] J. Liu, H.-D Yuan, X.-M. Lu, and X.-G. Wang, Quantum Fisher information matrix and multiparameter estimation, J. Phys. A: Math. Theor. 53, 023001 (2019). 
[40] W. Zhong, Z. Sun, J. Ma, X.-G. Wang, and F. Nori, Fisher information under decoherence in Bloch representation, Phys. Rev. A 87, 022337 (2013).

[41] J. A. Miszczak, Z. Puchała, P. Horodecki, A. Uhlmann, and K. Życzkowski, Sub- and super-fidelity as bounds for quantum fidelity, Quantum Inf. Comput. 9, 103 (2009).

[42] G. Balasubramanian, I. Y. Chan, R. Kolesov, M. Al-Hmoud, J. Tisler, C. Shin, C. Kim, A. Wojcik, P. R. Hemmer, A. Krueger, T. Hanke, A. Leitenstorfer, R. Bratschitsch, F. Jelezko, and J. Wrachtrup, Nanoscale imaging magnetometry with diamond spins under ambient conditions, Nature (London) 455, 648 (2008).

[43] J. R. Maze, P. L. Stanwix, J. S. Hodges, S. Hong, J. M. Taylor, P. Cappellaro, L. Jiang, M. V. Gurudev Dutt, E. Togan, A. S. Zibrov, A. Yacoby, R. L. Walsworth, and M. D. Lukin, Nanoscale magnetic sensing with an individual electronic spin in diamond, Nature (London) 455, 644 (2008).

[44] L. Rondin, J.-P. Tetienne, T. Hingant, J.-F. Roch, P. Maletinsky, and V. Jacques, Magnetometry with nitrogenvacancy defects in diamond, Rep. Prog. Phys. 77, 056503 (2014).

[45] D. Leibfried, E. Knill, S. Seidelin, J. Britton, R. B. Blakestad, J. Chiaverini, D. B. Hume, W. M. Itano, J. D. Jost, C. Langer et al., Creation of a six-atom 'Schrödinger cat' state, Nature (London) 438, 639 (2005).
[46] J. Zhang, G. Pagano, P. W. Hess, A. Kyprianidis, P. Becker, H. Kaplan, A. V. Gorshkov, Z.-X. Gong, and C. Monroe, Observation of a many-body dynamical phase transition with a 53-qubit quantum simulator, Nature (London) 551, 601 (2017).

[47] P. Jurcevic, H. Shen, P. Hauke, C. Maier, T. Brydges, C. Hempel, B. P. Lanyon, M. Heyl, R. Blatt, and C. F. Roos, Direct Observation of Dynamical Quantum Phase Transitions in an Interacting Many-Body System, Phys. Rev. Lett. 119, 080501 (2017).

[48] D. Gross, Y.-K. Liu, S. T. Flammia, S. Becker, and J. Eisert, Quantum State Tomography via Compressed Sensing, Phys. Rev. Lett. 105, 150401 (2010).

[49] A. Rath, C. Branciard, A. Minguzzi, and B. Vermersch, Quantum Fisher information from randomized measurements, arXiv:2105.13164.

[50] L. Isserlis, On a formula for the product-moment coefficient of any order of a normal frequency distribution in any number of variables, Biometrika 12, 134 (1918).

[51] B. Vermersch, A. Elben, M. Dalmonte, J. I. Cirac, and P. Zoller, Unitary $n$-designs via random quenches in atomic Hubbard and spin models: Application to the measurement of Rényi entropies, Phys. Rev. A 97, 023604 (2018).

[52] A. Elben, B. Vermersch, C. F. Roos, and P. Zoller, Statistical correlations between locally randomized measurements: A toolbox for probing entanglement in many-body quantum states, Phys. Rev. A 99, 052323 (2019). 